IAU Colloguium 164: Radio Emission from Galactic and Extragalactic Compact Sources

ASP Conference Series, Vol. 144,1998

J. A. Zensus, G. B. Taylor, \&f J.M. Wrobel (eds.)

\title{
VLBA Imaging of UX Ari
}

\author{
A. J. Beasley \& T.S. Bastian \\ National Radio Astronomy Observatory, Socorro, NM 87801, U.S.A.
}

Abstract. We have used the Very Long Baseline Array (VLBA) and the Very Large Array (VLA) to perform multi-wavelength radio imaging of the RSCVn active binary UX Arietis during the period November 16-21 1995. A series of strong flaring events exhibiting opticallythick spectra peaking above $100 \mathrm{GHz}$ and extended source structures consistent with interbinary emission were detected.

\section{Introduction \& Observations}

UX Ari is a member of the RS CVn class of active binaries which are characterized by orbital periods $\mathrm{P}_{\text {orb }} \sim 1-14$ days and greatly enhanced levels of activity (Hall 1976), including strong chromospheric emission, star spots, soft $\mathrm{X}$-ray emission, and flaring nonthermal radio emission. UX Ari is composed of a G5 dwarf and a K0 subgiant (Carlos \& Popper 1971). It has an orbital period of 6.44 days, an inclination of $\sim 60^{\circ}$, and a binary separation of $\sim 1.3 \times 10^{12}$ $\mathrm{cm}$. At a distance of $50 \mathrm{pc}$, this corresponds to a maximum angular separation of 1.7 mas at quadrature. UX Ari was observed with the VLBA during 1995 November 16-21, as part of an orbital monitoring campaign. Dual-polarization $32 \mathrm{MHz}$ bandwidths were recorded at observing frequencies ranging from 5$43 \mathrm{GHz}$; all observations were phase-referenced to the source $0326+277$, separation $1.1^{\circ}$. Contemporaneous observations were made of the microwave spectrum by the VLA. In Figure 1, the VLA spectra of the star is shown, including all data taken between 95Nov16 and 95Dec01. In Figure 2, a contour image of the 95 Nov17 data is shown, indicating a resolved source with dimensions similar to that expected from the binary separation at that epoch.

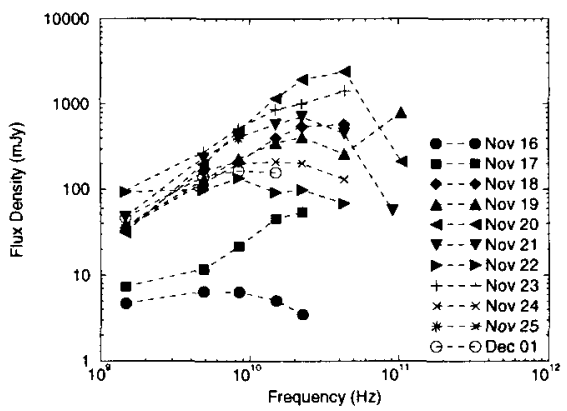

Figure 1. UX Ari VLA spectra: 95Nov16-95Dec01. $100 \mathrm{GHz}$ measurements from NRAO $12 \mathrm{~m}$ and Owens Valley Radio Observatory.

Several scenarios have been proposed to explain the flares on RS CVn binaries, including magnetic coupling between the stars (Uchida \& Sakurai 1983), or large loops anchored to one or both of the stars (Mutel et al. 1985, ChiuderiDrago \& Franciosini 1993). Our observations suggest that both stars likely 


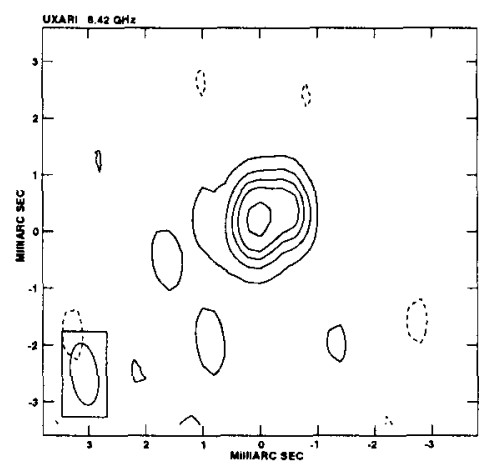

Figure 2. UX Ari VLBA image for 95 Nov17. Peak flux density $1.78 \mathrm{mJy}$, contour levels at $-1,2,4,6,8,12,16,32,64,128$ times $0.12 \mathrm{~m} \mathrm{Jy}$.

participate in the giant radio flares on UX Ari; we propose that the main site of the energy release is on the more active subgiant, where the magnetic field strength and electron number density are high, thereby producing a source which is self-absorbed to high radio frequencies. Estimates of the flare source size at UV and X-ray wavelengths (van den Oord et al. 1988, Tsuru et al. 1989) range from $5-10 \times 10^{10} \mathrm{~cm}$, suggesting these emissions are largely confined to the site of the flare. In some cases, however, due to coupling of the flare on subgiant with the magnetic field of the $G$ dwarf, a significant number of energetic electrons escape from the energy release site and fill the magnetized volume between the stars on a short timescale ( $\lesssim 1 \mathrm{~min}$ ). Hence, the radio flare involves a much larger volume than is involved in the UV or X-ray flare.

Acknowledgments. The National Radio Astronomy Observatory is a facility of the National Science Foundation, operated under a cooperative agreement by Associated Universities, Inc.

\section{References}

Carlos, R. C., \& Popper, D. M. 1971. PASP, 83, 504-507.

Chiuderi-Drago, F., \& Franciosini, E. 1993. ApJ, 410, 301-308.

Hall, D. S. 1976. IAU Colloq. 29, Multiple Periodic Variable Stars, ed. W. S. Fitch (Dordrecht: Reidel), pp. 287-290.

Mutel, R. L., et al. 1985. ApJ, 289, 262-268.

Tsuru, T., et al. 1989. PASJ, 41, 679-695.

Uchida, Y., \& Sakurai, T. 1983. Activity in Red Dwarf Stars, ed. P. B. Byrne, \& M. Rodonò (Dordrecht: Reidel), 629-632.

van den Oord, G. H. J., et al. 1988. $A \& A, \mathbf{2 0 5}, 181-196$. 